\title{
Dengue encephalitis in a 3-month-old para-neonate: a rare presentation of severe dengue
}

\author{
Vijay Sonawane, Veeranna Kotrashetti, Kapil Bainade, Vidisha Daiya*
}

Department of Paediatrics, D.Y. Patil University, School of Medicine, Nerul, Navi Mumbai, Maharashtra, India

Received: 03 December 2021

Revised: 30 December 2021

Accepted: 04 January 2022

\author{
*Correspondence: \\ Dr. Vidisha Daiya, \\ E-mail: vidishadaiya@gmail.com
}

Copyright: () the author(s), publisher and licensee Medip Academy. This is an open-access article distributed under the terms of the Creative Commons Attribution Non-Commercial License, which permits unrestricted non-commercial use, distribution, and reproduction in any medium, provided the original work is properly cited.

\begin{abstract}
Dengue fever is a common viral illness seen worldwide with a higher incidence in tropical regions. The illness may present as a mild illness with nonspecific symptoms to a life-threatening severe dengue. Severe dengue is usually seen in secondary dengue infection which is uncommon during infancy. As the dengue virus is classically not known to be a neurotropic virus, neurological manifestations are atypical presentations of severe dengue. The child may present with fever, seizures, altered mental status and focal neurological deficit. Diagnosis is confirmed in presence of clinical features with isolation of the virus in serum and/or cerebrospinal fluid. We present a case of a 3-month-old paraneonate who presented with fever, seizures and irritability without evidence of other causes of encephalitis with serum dengue NS1 and IgM positive and normal CSF routine analysis.
\end{abstract}

Keywords: Dengue fever, Seizures, Severe dengue, Dengue encephalitis

\section{INTRODUCTION}

Dengue fever is a common viral illness seen worldwide with a higher prevalence in the tropical countries. It has a broad spectrum of presentation ranging from asymptomatic to life threatening severe dengue which includes dengue shock, dengue hemorrhagic fever and multiorgan involvement. ${ }^{1}$

The dengue virus is not commonly known to be a neurotropic virus, although a few cases of dengue fever presenting with neurological manifestations have been reported.

Dengue encephalitis is an atypical and rare presentation and is classified as severe dengue. As severe dengue is more common with secondary dengue infection, it is rarely seen during infancy.

\section{CASE REPORT}

A 3-month-old para-neonate presented with acute history of fever for three days, excessive crying for one day and one episode of generalized tonic-clonic convulsion (GTCS), not associated with fever spike, which lasted for about 2 minutes. The child was developmentally normal for age, had no significant birth history or any prior history of convulsion. On examination, child was irritable, mild tachycardia and respiratory distress was present. On detailed examination, no signs of focal neurological deficit found, hepatomegaly was present. To evaluate for the cause, complete blood count and CRP (C-Reactive Protein) were sent which were within normal limits. Fundus examination and USG skull was done which were normal. CSF (cerebrospinal fluid) routine and culture done which was normal. Metabolic work up revealed hyponatremia (sodium $=116 \mathrm{mEq} / \mathrm{dL}$ ), correction for the same was given and repeat serum sodium had 
normalized, after which maintenance fluids were continued.

Child continued to have high grade fever spikes for which he was started on prophylactic antibiotics. On day 2 of admission, he had a second episode of GTCS convulsion and was loaded on injectable anti-epileptics. We repeated a complete blood count which showed thrombocytopenia and hemoconcentration. Dengue NS1 (non-structural protein-1) and antibodies were sent in view of clinical suspicion of severe dengue and high endemicity in our country. Dengue NS1 antigen and IgM antibodies both came positive. Diagnosis of dengue encephalitis was made as criteria were fulfilled. Neuroimaging could not be done due to financial constraints. We continued management with intravenous fluids, anti-epileptics and other symptomatic therapy. On further evaluation, repeat $\mathrm{CBC}$ showed improvement in hemoconcentration and normalization of platelet counts. Child became afebrile, gradually stabilized and did not develop any further complications of dengue fever and recovered well.

\section{DISCUSSION}

Dengue is an arthropod-borne viral infection caused by the dengue virus belonging to the flaviviridae family. The disease is transmitted by the bite of an infected Aedes Aegypti mosquito. After an incubation period of 3-14 days, the disease may develop with a varying presentation ranging from mild illness to life threatening severe dengue. The World Health Organization (WHO) has classified the disease based on its features into dengue without warning signs, dengue with warning signs and severe dengue. ${ }^{1}$ The course of dengue illness generally follows 3 phases: febrile phase, critical phase and recovery phase. ${ }^{1}$ Children with mild illness may not have the critical phase.

Classically, the dengue virus is a non-neurotropic virus, but some cases of neurological complications have been reported. The virus has four serotypes DENV-1, DENV2, DENV-3 and DENV-4, out of which DENV-2 and DENV-3 serotypes are more commonly associated with neurological complications. ${ }^{2}$ Neurological manifestation of dengue was first reported in 1976 in Thailand as an atypical presentation of dengue. ${ }^{3}$ In recent years, the incidence was found to be varying from 0.5 to $20 \% .^{4,5}$ As per the latest classification, neurological manifestations classify as severe dengue. ${ }^{6}$ Severe dengue is usually seen in secondary dengue infection, hence it is usually seen in older children. Severe dengue is uncommon in young children, especially during neonatal and para-neonatal period. One neonate with dengue encephalitis has been reported so far.

The pathogenesis of neurological manifestations is not clearly understood but is most likely associated with direct invasion of the central nervous system by the virus, autoimmune reactions and metabolic alterations. ${ }^{6}$ Like in our case, it was associated with severe hyponatremia. Host immunity, age and genetic makeup also play a role in severity of the infection. ${ }^{6}$

Neurological manifestations of dengue have been classified by Murthy into 3 categories: manifestations due to neurotropic effects which includes encephalitis, meningitis, myositis and myelitis. Systemic complications resulting in encephalopathy, stroke and hypokalemic transient paralysis. Post-infectious complications which include Guillain-Barre syndrome, optic neuritis and encephalomyelitis. ${ }^{7}$ The classical symptoms of dengue such as fever, myalgia, gastrointestinal symptoms, bleeding manifestations and rash may not be seen in these patients.

The criteria for dengue encephalitis includes fever, acute signs of cerebral involvement (seizures, altered mental status), presence of dengue IgM antibodies or genomic material in serum and/or CSF and ruling out other causes of encephalitis. ${ }^{8}$ Our patient had fever, seizures and presence of dengue IgM antibodies with other common causes being ruled, thus fulfilling the criteria. Isolation of dengue virus in CSF has poor sensitivity as seen in a study conducted by Solomon et al on 9 patients where the virus could be isolated in CSF from only 2 patients. These patients had serum confirmed diagnosis with CSF pleocytosis or seizures or focal neurological signs with most of them not having typical features of dengue fever. Hence, in conclusion, dengue encephalitis should be suspected in patients on the basis of such clinical presentation living in endemic areas. ${ }^{9}$

Neuroimaging in dengue encephalitis may show variable findings. Most patients have normal neuroimaging study. Abnormal findings if present are nonspecific and include cerebral oedema and focal changes in basal ganglia, hippocampus and thalamus. ${ }^{10}$ In our patient, CT or MRI brain was not done due to financial constraints, although, an ultrasonogram of the skull was done which was normal.

Treatment of dengue encephalitis is mainly supportive. Fluids, anti-epileptics and other symptomatic therapy as indicated is recommended. Recovery and prognosis of most patients with dengue encephalitis is good.

\section{CONCLUSION}

Dengue encephalitis is a rare and atypical presentation of severe dengue. As severe dengue is more common with secondary dengue infection, it is rarely found in younger children, especially during para-neonatal period and infancy. In highly endemic areas, children presenting with features of encephalitis, dengue encephalitis should be considered in presence of clinical suspicion. Treatment is mainly supportive requiring fluid resuscitation and anti-epileptics as indicated. Recovery and prognosis of most children with dengue encephalitis is good. 
Funding: No funding sources

Conflict of interest: None declared

Ethical approval: Not required

\section{REFERENCES}

1. World Health Organization. Dengue: guidelines for diagnosis, treatment, prevention and control. Geneva, Switzerland: WHO. 2009.

2. Varatharaj A. Encephalitis in the clinical spectrum of dengue infection. Neurol India. 2010;58(4):58591.

3. Sanguansermsri T, Poneprasert B, Phornphutkul B. Acute encephalopathy associated with dengue infection. Bangkok, Thailand. Seameo Tropmed. 1976;10-11.

4. Murthy JM. Neurological complication of dengue infection. Neurol India. 2010;58:581-4.

5. Sahu R, Verma R, Jain A, Garg RK, Singh MK, Malhotra HS, et al. Neurologic complications in dengue virus infection: a prospective cohort study. Neurology. 2014;83:1601-9.
6. Li GH, Ning ZJ, Liu YM, Li XH. Neurological Manifestations of Dengue Infection. Front. Cell. Infect. Microbiol. 2017;7:449.

7. Murthy JM. Neurological complication of dengue infection. Neurol India. 2010;58:581-4.

8. Soares C, Puccioni-Sohler M. Dengue encephalitis: suggestion for case definition. $J$ Neurol Sci. 2011;306:165.

9. Solomon T, Dung NM, Vaughn DW, Kneen R, Thao LT, Raengsakulrach B, et al. Neurological manifestations of dengue infection. Lancet. 2000;355:1053-9.

10. Bhoi SK, Naik S, Kumar S, Phadke RV, Kalita J, Misra UK. Cranial imaging findings in dengue virus infection. J Neurol Sci. 2014;342:36-41.

Cite this article as: Sonawane V, Kotrashetti V, Bainade K, Daiya V. Dengue encephalitis in a 3month-old para-neonate: a rare presentation of severe dengue. Int J Contemp Pediatr 2022;9:208-10. 